\title{
THE ASYMPTOTICS OF THE DETERMINANT FUNCTION FOR A CLASS OF OPERATORS
}

\author{
LEONID FRIEDLANDER
}

(Communicated by Paul S. Muhly)

\begin{abstract}
Let $A$ be an elliptic pseudodifferential operator on a closed manifold $M$ and ord $A>\operatorname{dim} M$. We derive the asymptotics of $\log \operatorname{det}\left(1+\varepsilon A^{-1}\right)$ when $\varepsilon \rightarrow \infty$. The constant term of this asymptotics equals $-\log \operatorname{det} A$.
\end{abstract}

\section{INTRODUCTION}

Let $A$ be an elliptic pseudodifferential operator on a closed manifold $M$ and $n=\operatorname{ord} A>\operatorname{dim} M=d$. Suppose that $\arg (A \varphi, \varphi) \leq \alpha, 0 \leq \alpha<\pi / 2$. One can define complex powers of the $A$ and $A^{-s}$ are operators from the trace class when $\operatorname{Re} s>d / n$. The $\zeta$-function $\zeta(s)=\operatorname{Tr} A^{-s}$ is holomorphic in the half-plane $\operatorname{Re} s>d / n$ and admits analytic continuation to a meromorphic function in the whole complex plane. The poles of this function are located in the points $(d-j) / n ; j=0,1,2, \ldots$. The point 0 is not really a pole of $\zeta(s)$. If the operator $A$ is differential or it is a power of a differential operator some other poles drop. The residues of $\zeta(s)$ and the numbers $\zeta(0), \zeta(-1), \ldots$ can be calculated if one knows the complete symbol of the operator $A$ [1-3]. Particularly the point $s=0$ is a regular point of the $\zeta$-function and one can define

$$
W(A)=\log \operatorname{det} A=-\zeta^{\prime}(0) .
$$

It is easy to check that this definition gives us the generalization of a finite dimensional operator's determinant.

On the other hand the inverse operator $A^{-1}$ belongs to the trace class and the determinants

$$
D(\varepsilon)=\operatorname{det}\left(1+\varepsilon A^{-1}\right)=\prod_{j=1}^{\infty}\left(1+\varepsilon \lambda_{j}\right) \quad\left(\lambda_{j}=\mu_{j}^{-1}\right)
$$

Received by the editors November 14, 1988.

1980 Mathematics Subject Classification (1985 Revision). Primary 47G05; Secondary 47B25, 47B38. 
are defined. The infinite product in the right hand side of (2) converges. It is more convenient to consider the function

$$
d(\varepsilon)=\log D(\varepsilon)=\sum_{j=1}^{\infty} \log \left(1+\varepsilon \lambda_{j}\right) .
$$

We are going to investigate the asymptotic expansion of the $d(\varepsilon)$ when $\varepsilon \rightarrow$ $+\infty$. In particular the determinant $W(A)$ will appear in this expansion.

We think there are two reasons why this expansion is interesting. The first reason is the connection between the Fredholm determinant $\operatorname{det}\left(1+\varepsilon A^{-1}\right)$ and the determinant of the elliptic operator $A$ we shall obtain from this expansion. The second reason is that the value $\operatorname{det}\left(1+\varepsilon A^{-1}\right)$ appears in the measure theory as the result of integration of $\exp (-\varepsilon(x, x) / 2)$ over a Hilbert space with respect to a Gaussian measure with average 0 and correlation operator $A^{-1}$ (e.g. see [4]).

The fact that $\operatorname{det}\left(1+\varepsilon A^{-1}\right)$ admits asymptotic expansion for big $\varepsilon$ is not surprising. Let us differentiate formally the equality (3) with respect to $\varepsilon$ :

$$
d^{\prime}(\varepsilon)=\sum_{j=1}^{\infty} \lambda_{j}\left(1+\varepsilon \lambda_{j}\right)^{-1}=\sum_{j=1}^{\infty}\left(\mu_{j}+\varepsilon\right)^{-1}=\operatorname{Tr}(A+\varepsilon)^{-1} .
$$

The trace of the resolvent $\operatorname{Tr}(A+\varepsilon)^{-1}$ admits asymptotic expansion when $\varepsilon \rightarrow \infty$ [5]. So one can justify the possibility of the last expansion's integration and receive the asymptotics for $d(\varepsilon)$. The only thing we can not get in this way is the constant of integration, i.e. the term with $\varepsilon^{0}$. We shall see later that this term is of the most interest. So we shall derive the asymptotics for $d(\varepsilon)$ directly.

The aim of this paper is to prove

Theorem. The function $d(\varepsilon)$ admits asymptotic expansion

$$
d(\varepsilon) \sim \sum_{k=-d}^{\infty} p_{k} \varepsilon^{-k / n}+\sum_{j=0}^{\infty} q_{j} \varepsilon^{-j} \log \varepsilon
$$

with

$$
p_{0}=-\log \operatorname{det} A \text {. }
$$

\section{CONNECTION BETWEEN $d(\varepsilon)$ AND $\zeta(\varepsilon)$}

In this section we shall prove

Proposition 1. Let $d / n<a<1$. Then

$$
d(\varepsilon)=\frac{1}{2 \pi i} \int_{\operatorname{Re} s=a} \varepsilon^{s} b(s) \zeta(s) d s
$$

where

$$
b(s)=\frac{1}{s} \int_{0}^{\infty} \frac{t^{-s}}{1+t} d t .
$$


Proof. It is convenient to introduce the new argument $\omega=\log \varepsilon$. Let $d^{*}(\varepsilon)=$ $d\left(e^{\omega}\right)$. Then formula (6) can be rewritten in the form

$$
d^{*}(\omega)=\frac{1}{2 \pi i} \int_{\operatorname{Re} s=a} e^{s \omega} b(s) \zeta(s) d s .
$$

Integrating by parts and changing the argument $t=e^{\omega}$ we derive

$$
b(s)=\int_{0}^{\infty} t^{-s-1} \log (1+t) d t=\int_{-\infty}^{\infty} e^{-\omega s} \log \left(1+e^{\omega}\right) d \omega .
$$

Note that the last formula gives the Fourier transformation $\log \left(1+e^{\omega}\right) \rightarrow b(i s)$. So by the inverse Fourier formula

$$
\log \left(1+e^{\omega}\right)=\frac{1}{2 \pi i} \int_{\operatorname{Re} s=a} b(s) e^{\omega s} d s .
$$

After substituting $\varepsilon \lambda$ instead of $e^{\omega}$ we derive

$$
\log (1+\varepsilon \lambda)=\frac{1}{2 \pi i} \int_{\operatorname{Re} s=a} \varepsilon^{s} b(s) \lambda^{s} d s .
$$

Now to obtain (6) we have to sum the last identities for $\lambda=\lambda_{j}$. Note that the summation and integration operations in the right hand side commute and $\zeta(s)$ is the sum of $\lambda_{j}^{s}$.

\section{ANALYTICAL PROPERTIES OF $b(s)$}

We shall obtain asymptotic expansion for $d^{*}(\omega)$ (or $d(\omega)$ ) by shifting the contour in (8) to the left. So we must have analytical continuation of $b(s)$ into the left half-plane and we must estimate $|b(s)|$ and $|\zeta(s)|$ for large $|\operatorname{Im} s|$. In this section we shall prove

Proposition 2. The function $b(s)$ admits analytical continuation to a meromorphic function into the half-plane $\operatorname{Re} s<1$. It has a pole of order 2 into the point $s=0$ and simple poles into the points $-1,-2, \ldots ; \operatorname{Res}_{s=0} b(s)=0$, $\operatorname{Res}_{s=0} s b(s)=1$. The function $b(s)$ satisfies the following estimate

$$
|b(\sigma+i \tau)| \leq C(\sigma)|\tau|^{\sigma-1} \exp \left(-\frac{\pi}{2}|\tau|\right), \quad \sigma<1,|\tau| \geq 1 .
$$

Proof. Let

$$
J_{n}=\int_{0}^{\infty} t^{-s}(1+t)^{n} d t, \quad n \geq 1 .
$$

Integrating by parts we obtain

$$
J_{n}=-\frac{n}{s-1} \int_{0}^{\infty} \frac{t^{1-s}}{(1+t)^{n+1}} d t=-\frac{n}{s-1} J_{n}+\frac{n}{s-1} J_{n+1} .
$$

So

$$
\frac{s+n-1}{s-1} J_{n}=\frac{n}{s-1} J_{n+1}
$$


and

$$
J_{n}=\frac{n}{s+n-1} J_{n+1}
$$

Thus

$$
J_{1}=\frac{k !}{s(s+1) \cdots(s+k-1)} \int_{0}^{\infty} t^{-s}(1+t)^{-k-1} d t .
$$

The integral in the right hand side of $(10)$ is absolutely convergent in the strip $-k<\operatorname{Re} s<1$. So the assertion about analytical continuation of $b(s)$ is proved.

Applying (10) with $k=1$ we get

$$
\begin{aligned}
\operatorname{Res}_{s=0} s b(s) & =\int_{0}^{\infty} \frac{d t}{(1+t)^{2}}=1 ; \\
\operatorname{Res}_{s=0} b(s) & =\left.\frac{d}{d s} \int_{0}^{\infty} \frac{t^{-s}}{(1+t)^{2}} d t\right|_{s=0}=-\int_{0}^{\infty} \frac{\log t}{(1+t)^{2}} d t .
\end{aligned}
$$

Changing the argument $t \rightarrow 1 / u$ in the last integral one can see that

$$
\int_{0}^{\infty} \frac{\log t}{(1+t)^{2}} d t=-\int_{0}^{\infty} \frac{\log u}{(1+u)^{2}} d \dot{u}
$$

so

$$
\operatorname{Res}_{s=0} b(s)=0 .
$$

To prove (9) we shall use the representation (10). The number $k$ in (10) will be large for large $|\tau|$ (really $k \sim|\tau|^{2}$ ). Suppose that $-\infty<\sigma_{0}<\sigma<\sigma_{1}<1$ with some $\sigma_{0}$ and $\sigma_{1}$. All constants in the following estimates will depend on $\sigma_{0}$ and $\sigma_{1}$ only. We shall not numerate these constants. They will be designated by the same letter $C$.

To begin with, let us estimate the integral (10). This integral splits into

$$
J=J_{1}+J_{2}=\int_{0}^{1 / k} t^{-s}(1+t)^{-k-1} d t+\int_{1 / k}^{\infty} t^{-s}(1+t)^{-k-1} d t .
$$

The estimation of $J_{1}$ is very easy:

$$
\left|J_{1}\right| \leq C \int_{0}^{1 / k} t^{-\sigma} d t \leq C k^{\sigma-1} .
$$

To estimate $J_{2}$ let us suppose that $0 \leq \sigma \leq 1$ (note that we shift $s$ from the strip $\sigma_{0}<\sigma<\sigma_{1}$ !). The function $t^{-\sigma}$ decreases, so

$$
\left|J_{2}\right| \leq k^{\sigma} \int_{1 / k}^{\infty}(1+t)^{-(k+1)} d t \leq k^{\sigma-1} .
$$


If $\sigma<0$ we shall integrate by parts $n=|[\sigma]|$ times $([x]$ —entire part of the number $x$ ).

$$
\begin{aligned}
\left|J_{2}\right| \leq & \int_{1 / k}^{\infty} t^{-\sigma}(1+t)^{-k-1} d t \\
= & k^{\sigma-1}\left(1+\frac{1}{k}\right)^{k}-\frac{\sigma}{k} \int_{1 / k}^{\infty} t^{-\sigma-1}(1+t)^{-k} d t=\cdots \\
= & k^{\sigma-1}\left(1+\frac{1}{k}\right)^{-k}-\sigma \frac{k^{\sigma}}{k-1}\left(1+\frac{1}{k}\right)^{-(k-1)}+\cdots \\
& +(-1)^{n} \frac{\sigma(\sigma+1) \cdots(\sigma+n-1)}{k(k-1) \cdots(k-n+1)} \int_{1 / k}^{\infty} t^{-f r(\sigma)}(1+t)^{-k+n-1} d t .
\end{aligned}
$$

Every term except the last is obviously estimated by $C k^{\sigma-1}$. The integral in the last term is the integral of the form we have just investigated $(k \mapsto k-n$; $\sigma \mapsto f r(\sigma))$, so it is estimated by $k^{f r(\sigma)-1}(f r(\sigma)=\sigma-[\sigma])$. The product which stands before the integral is of the order $k^{-n}=k^{-[\sigma]}$. Thus the last term is also estimated by $k^{\sigma-1}$. Finally,

$$
\left|J_{2}\right| \leq C k^{\sigma-1}
$$

and

$$
\left|\int_{0}^{\infty} t^{-s}(1+t)^{-k-1} d t\right| \leq C k^{\sigma-1} .
$$

Now we intend to estimate the product which stands before the integral in the right hand side of (10). Denote

$$
r(k)=\log \left|\frac{k !}{s^{2}(s+1) \cdots(s+k-1)}\right|
$$

and

$$
l=\left|\left[\sigma_{0}\right]\right|+1 .
$$

We have

$$
\begin{aligned}
r(k)= & \log k !-2 \log |s|-\sum_{j=1}^{l-1} \log |s+j|-\sum_{j=l}^{k-1} \log |s+j| \\
\leq & \log k !-(l+1) \log |\tau|-\frac{1}{2} \sum_{j=1}^{k-1} \log \left((\sigma+j)^{2}+\tau^{2}\right) \\
= & \log k !-(l+1) \log |\tau|-\sum_{j=1}^{k-1} \log (\sigma+j)-\frac{1}{2} \sum_{j=l}^{k-1} \log \left(1+\frac{\tau^{2}}{(\sigma+j)^{2}}\right) \\
= & \log l !+\sum_{j=0}^{k-l-1} \log \left(\frac{k-j}{k-j+\sigma-1}\right)-\frac{1}{2} \sum_{j=l}^{k-1} \log \left(1+\frac{\tau^{2}}{(\sigma+j)^{2}}\right) \\
& -(l+1) \log |\tau| .
\end{aligned}
$$


The first term in the right hand side of (12) is constant. The second term is bounded by $C+(1-\sigma) \log k$. Indeed,

$$
\begin{aligned}
\sum_{j=0}^{k-1} \log \left(\frac{k-j}{k-j+\sigma-1}\right)= & \sum_{j=0}^{k-l-1} \log \left(1+\frac{1-\sigma}{k-j+\sigma-1}\right) \\
= & \sum_{j=0}^{k-l-1} \log \left(1+\frac{1-\sigma}{l+\sigma+j}\right) \\
\leq & \log \left(1+\frac{1-\sigma}{l+\sigma}\right)+\int_{1}^{k-l} \log \left(1+\frac{1-\sigma}{x}\right) d x \\
= & C+\left.x \log \left(1+\frac{1-\sigma}{x}\right)\right|_{1} ^{k-l} \\
& +(1-\sigma) \int_{1}^{k-l} \frac{d x}{(x+1-\sigma)} \\
\leq & C+(1-\sigma) \log k .
\end{aligned}
$$

To estimate the third term in the right hand side of (12) we note that the function $\log \left(1+\tau^{2} /(\sigma+j)^{2}\right)$ is decreasing with respect to $j$. Thus

$$
\begin{aligned}
\frac{1}{2} \sum_{j=l}^{k-1} \log \left(1+\frac{\tau^{2}}{(\sigma+j)^{2}}\right) \geq & \frac{1}{2} \int_{l}^{k} \log \left(1+\frac{\tau^{2}}{(\sigma+x)^{2}}\right) d x \\
= & \frac{1}{2} \int_{l+\sigma}^{k+\sigma} \log \left(1+\frac{\tau^{2}}{x^{2}}\right) d x \\
= & \frac{1}{2}(k+\sigma) \log \left(1+\frac{\tau^{2}}{(\sigma+k)^{2}}\right) \\
& -\frac{1}{2}(l+\sigma) \log \left(1+\frac{\tau^{2}}{(\sigma+l)^{2}}\right) \\
& +\tau \arctan \frac{k+\sigma}{\tau}-\tau \arctan \frac{l+\sigma}{\tau} \\
\geq & C+\frac{1}{2}(k+\sigma) \log \left(1+\frac{\tau^{2}}{(\sigma+k)^{2}}\right)-(l+\sigma) \log |\tau| \\
& +|\tau| \arctan \frac{k+\sigma}{|\tau|} .
\end{aligned}
$$


Finally

$$
\begin{aligned}
r(k) \leq & C+(1+\sigma) \log k-\frac{1}{2}(k+\sigma) \log \left(1+\frac{\tau^{2}}{(\sigma+k)^{2}}\right) \\
& +(l+\sigma) \log |\tau|-|\tau| \arctan \frac{k+\sigma}{|\tau|}-(l+1) \log |\tau| \\
= & C+(1-\sigma) \log k+(\sigma-1) \log |\tau| \\
& -\frac{1}{2}(k+\sigma) \log \left(1+\frac{\tau^{2}}{(\sigma+k)^{2}}\right)-|\tau| \arctan \frac{k+\sigma}{|\tau|} .
\end{aligned}
$$

Now we take $k \sim \tau^{2}$. Then the second term in the right hand side of (13) equals $2(1-\sigma) \log |\tau|$ up to the additive constant. The fourth term is bounded. The fifth term equals

$$
|\tau| \arctan (|\tau|+o(1))=\frac{\pi}{2}|\tau|+o(1) .
$$

So

$$
r(k) \leq-\frac{\pi}{2}|\tau|+(1-\sigma) \log |\tau|+C ; k \sim \tau^{2} .
$$

Therefore after substitution $\left[\tau^{2}\right]$ instead of $k$ into (11) we obtain

$$
|b(s)| \leq C|\tau|^{2(\sigma-1)} \exp \left(-\frac{\pi}{2}|\tau|+(1-\sigma) \log |\tau|\right)=C|\tau|^{\sigma-1} \exp \left(-\frac{\pi}{2}|\tau|\right) .
$$

4. Estimation OF $|\zeta(\sigma+i \tau)|$ FOR LARGE $|\tau|$

Proposition 3. If operator $A$ satisfies the assumptions of this paper then

$$
|\zeta(\sigma+i \tau)| \leq C|\tau|^{-\sigma-1 / 2} \exp \left(\frac{\pi}{2}|\tau|\right), \quad C=C(\sigma),|\tau| \geq 1 .
$$

Proof. We shall use the representation

$$
\zeta(s)=\frac{1}{\Gamma(s)} \int_{0}^{\infty} t^{s-1} \theta(t) d t=\frac{J(s)}{\Gamma(s)}
$$

with

$$
\theta(t)=\operatorname{Tr} e^{-t A}=\sum_{j=1}^{\infty} e^{-t \mu_{j}}
$$

It is well known (e.g. see [2]) that

$$
\theta(t) \sim e^{-a t}, \quad t \rightarrow \infty
$$

and (Minakshisundaram-Plejel expansion)

$$
\theta(t) \sim \sum_{j=-d}^{0} a_{j} t^{j / n}+\sum_{j=1}^{\infty}\left(a_{j} t^{j / n}+b_{j} t^{j / n} \log t\right), \quad t \rightarrow 0
$$


Moreover one can differentiate both expansions (17) and (18). Split the integral from the right hand side of (15):

$$
J(s)=J_{1}(s)+J_{2}(s)=\int_{0}^{1} t^{s-1} \theta(t) d t+\int_{1}^{\infty} t^{s-1} \theta(t) d t .
$$

To estimate $J_{2}$ we use partial integration

$$
\begin{aligned}
\left|J_{2}(s)\right| & =\left|\frac{\theta(1)}{s}+\frac{1}{s} \int_{1}^{\infty} t^{s} \theta^{\prime}(t) d t\right| \leq \frac{1}{|s|}\left[\theta(1)+C \int_{1}^{\infty} t^{\sigma} e^{-a t} d t\right] \\
& \leq \frac{C}{|\tau|} ; \quad C=C(\sigma) .
\end{aligned}
$$

To estimate $J_{1}$ we take $l=1+|[\sigma]|$. Then

$$
\theta(t)=\sum_{j=-d}^{l}\left(a_{j} t^{j / n}+b_{j} t^{j / n} \log t\right)+r(t)
$$

$\left(b_{j}=0\right.$ if $\left.j \leq 0\right)$ with

$$
|r(t)| \leq C t^{(l+1) / n}|\log t| \quad \text { and } \quad\left|r^{\prime}(t)\right| \leq C t^{l / n}|\log t| ; \quad 0 \leq t \leq 1 .
$$

So

$$
\begin{aligned}
J_{1}(s) & =\sum_{j=-d}^{l} a_{j} \int_{0}^{1} t^{j / n+s-1} d t+\sum_{j=1}^{l} b_{j} \int_{0}^{1} t^{j / n+s-1} \log t d t+\int_{0}^{1} t^{s-1} r(t) d t \\
& =\sum_{j=-d}^{l} \frac{a_{j}}{s+j / n}-\sum_{j=1}^{l} \frac{b_{j}}{(s+j / n)^{2}}+\frac{1}{s} r(1)-\frac{1}{s} \int_{0}^{1} t^{s} r^{\prime}(t) d t
\end{aligned}
$$

and

$$
\left|J_{1}(s)\right| \leq C /|s| \leq C /|\tau|
$$

Finally

$$
|J(s)| \leq \frac{C}{|\tau|}, \quad C=C(\sigma) .
$$

To estimate $1 / \Gamma(s)$ we use the Stirling asymptotics $\log \Gamma(s)=(z-1 / 2) \log z-z+(1 / 2) \log (2 \pi)+o(1) ; \quad|z| \rightarrow \infty,|\arg z|<\pi$ (e.g. see [6]). We can write

$$
\begin{aligned}
\log \Gamma(\sigma+i \tau)= & {[(\sigma-1 / 2)+i \tau]\left[(1 / 2) \log \left(\sigma^{2}+\tau^{2}\right)+i \arg (\sigma+i \tau)\right] } \\
& -\sigma-i \tau+(1 / 2) \log (2 \pi)+o(1)
\end{aligned}
$$

and

$|\Gamma(\sigma+i \tau)| \sim \sqrt{2 \pi} e^{-\sigma} \exp \left((1 / 2)(\sigma-1 / 2) \log \left(\sigma^{2}+\tau^{2}\right)-\tau \arg (\sigma+i \tau)\right) ; \quad|\tau| \rightarrow \infty$.

Note that

$$
\arg (\sigma+i \tau)=\frac{\pi}{2} \operatorname{sgn} \tau-\frac{\sigma}{\tau}+o\left(\frac{1}{|\tau|}\right)
$$


and

Therefore

$$
\log \left(\sigma^{2}+\tau^{2}\right)=2 \log |\tau|+o\left(1 /|\tau|^{2}\right)
$$

$$
|\Gamma(\sigma+i \tau)| \sim \sqrt{2 \pi}|\tau|^{\sigma-1 / 2} e^{-\pi|\tau| / 2} ; \quad|\tau| \rightarrow \infty .
$$

Now the assertion of Proposition 3 follows from (15), (19) and (20).

\section{PROOF OF THE THEOREM}

Propositions 2 and 3 show us that the function which is integrated in (8) is bounded by $C|\tau|^{-3 / 2}$. So we can shift the path of integration to the left in the complex plane as far as we want. The poles of the function $b(s) \zeta(s)$ give us terms in asymptotics of $d^{*}(\omega)$. Denote

$$
\begin{aligned}
& \beta_{j}=\left.\operatorname{Res} b(s)\right|_{s=-j}, \quad j=0,1, \ldots ; \quad \hat{\beta}_{0}=\left.\operatorname{Res} s b(s)\right|_{s=0} ; \\
& \hat{\beta}_{j}=\left.\left(b(s)-\beta_{j} /(s+j)\right)\right|_{s=-j}, \quad j=1,2, \ldots ; \\
& \alpha_{k}=\left.\operatorname{Res} \zeta(s)\right|_{s=-k / n}, \quad k=-d,-d+1, \ldots ; \\
& \gamma_{j}=\left.\left(\zeta(s)-\alpha_{n j} /(s+j)\right)\right|_{s=-j}, \quad j=1,2, \ldots .
\end{aligned}
$$

Note that $\alpha_{0}=0$ and if $A$ is a differential operator or it is a power of a differential operator then

By Proposition 2

$$
\alpha_{n j}=0 \quad \text { and } \quad \gamma_{j}=\zeta(-j)
$$

$$
\beta_{0}=0 \text { and } \hat{\beta}_{0}=1 .
$$

The points $s=-k / n, k \neq j n(j=0,1, \ldots)$ are simple poles of the function

$$
F(s)=e^{s \omega} b(s) \zeta(s)
$$

and

$$
\operatorname{Res}_{s=-k / n} F(s)=\alpha_{k} e^{-k \omega / n} b(-k / n) .
$$

The points $s=j n$ are poles of the second order of $F(s)$. It is easy to calculate residues in these points:

$$
\begin{aligned}
\operatorname{Res}_{s=0} F(s) & =\zeta^{\prime}(0)+\omega \zeta(0), \\
\operatorname{Res}_{s=-j} F(s) & =\alpha_{n j} \beta_{j} \omega e^{-j \omega}+\left(\alpha_{n j} \hat{\beta}_{j}+\gamma_{j} \beta_{j}\right) e^{-j \omega} .
\end{aligned}
$$

Thus we have obtained

$$
d^{*}(\omega) \sim \sum_{k=-d}^{\infty} p_{k} e^{-k \omega / n}+\sum_{j=0}^{\infty} q_{j} \omega e^{-j \omega}, \quad \omega \rightarrow \infty
$$

with

$$
\begin{aligned}
p_{k} & =\alpha_{k} b(-k / n), k \neq j n(j=0,1, \ldots) ; \\
p_{0} & =\zeta^{\prime}(0) \\
p_{j n} & =\alpha_{n j} \hat{\beta}_{j}+\gamma_{j} \beta_{j}, \quad j=1,2, \ldots ; \\
q_{0} & =\zeta(0) ; q_{j}=\alpha_{n j} \beta_{j} .
\end{aligned}
$$

After substituting $e^{-\omega}=\varepsilon$ into (22) one obtains (4). In particular (5) holds. 


\section{REFERENCES}

1. R. T. Seeley, Complex powers of an elliptic operator, Proc. Sympos. Pure Math. 10, Amer. Math. Soc., 1967, 288-307.

2. M. E. Taylor, Pseudodifferential operators, Princeton University Press, Princeton, NJ, 1981.

3. M. A. Shubin, Pseudodifferential operators and spectral theory, Springer-Verlag, BerlinHeidelberg-New York, 1987.

4. N. N. Vakhania, Probability distributions on linear spaces, North Holland, New York-Oxford, 1981.

5. M. S. Agranovich, Some asymptotic formulas for elliptic pseudodifferential operators, Funct. Anal. and its Appl., 21 (1987) 53-56.

6. H. Bateman and A. Erdelyi, Higher transcendental functions, Vol. 1, McGraw-Hill Comp. Inc., New York-Toronto-London, 1953.

Department of Mathematics, Massachusetts Institute of Technology, Cambridge, MASSACHUSETTS 02139 